\title{
Mental Health Literacy: a Critical Target for Narrowing Racial Disparities in Behavioral Health
}

\author{
R. R. Tambling ${ }^{1} \cdot$ C. D'Aniello ${ }^{2} \cdot$ B. S. Russell ${ }^{1}$
}

Accepted: 21 October 2021

(C) The Author(s), under exclusive licence to Springer Science+Business Media, LLC, part of Springer Nature 2021

\begin{abstract}
One of the most persistent and troubling health disparities is the underutilization of mental health services, particularly for depression and anxiety, commonly occurring behavioral health concerns. The gap between individuals who need mental health care and those who receive care is large, and identified barriers to treatment include poor mental health and insurance literacy, as well as stigmatizing attitudes toward mental health disorders. The present study presents the results of an inquiry into the mental health literacy, insurance literacy, internalized stigma, and mental health symptoms. Results suggest that mental health literacy is poor and associated with higher rates of depression, anxiety, stress, internalized stigma, and caregiver burden. Implications of poor mental health literacy, as well as demographic disparities in literacy, are discussed.
\end{abstract}

Keywords Mental health literacy · Health insurance literacy $\cdot$ Internalized stigma · Behavioral health disparities $\cdot$ Mental illness

One of the most persistent and troubling health disparities is the underutilization of mental health services and the failure to engage in early intervention and prevention for behavioral health concerns. Depression and anxiety disorders are the most prevalent mental health disorders, with lifetime prevalence rates of $17 \%$ for depression and $29 \%$ for anxiety disorders (Kessler et al, 2002, 2012). The prevalent nature of depression and anxiety carries steep costs to individuals, families, and society. Depression and anxiety are the $1^{\text {st }}$ and $6^{\text {th }}$ largest contributors to global disability, respectively (Roll et al., 2013). The gap between individuals who need mental health care and those who receive care is staggering, as up to $70 \%$ of individuals who need mental health treatment fail to access the services they need (Thornicroft, 2007). Barriers to treatment include lack of access to services, the potential client's limited overall understanding of therapy treatment, and the perception that therapy treatment is too demanding or not relevant to the presenting concerns. These barriers are

R. R. Tambling

rachel.tambling@uconn.edu

1 Department of Human Development \& Family Sciences, University of Connecticut, 348 Mansfield Road, Unit 1058, Storrs, CT 06269-1058, USA

2 Department of Community, Family, and Addiction Services, Texas Tech University, Lubbock, TX, USA 
directly related to mental health literacy (MHL; Jorm, 2015), a direct driver of the gap between treatment need and utilization. Crucial information is lacking about vulnerable groups' experiences of stress and burden specific to the role of MHL and stigma and the adaptive coping required to protect mental health and experience positive behavioral health outcomes. In the present study, researchers sought to provide this information, assessing mental health literacy, mental health, and health insurance literacy across a diverse group of Americans during the COVID-19 global pandemic, a disaster impacting mental health and wellness.

\section{Background}

MHL refers to knowledge and beliefs about mental disorders with respect to recognition, management, and prevention. Individuals with higher levels of mental health literacy recognize mental health problems and symptoms, know where to seek help and information, and understand that the mental health problems can and should be treated. Mental health literacy is associated with behavioral health care utilization (Mendenhall and Frauenholtz, 2013) and propensity to seek behavioral health services (Smith \& Shochet, 2011). Unfortunately, mental health literacy in the general US public is low (Dunn et al., 2009), and most individuals struggle to identify mental health disorders and are uncertain how to obtain information and treatment if they encounter difficulties (Jorm et al., 2006). In particular, individuals have a particularly difficult time identifying problematic symptoms of anxiety (Coles \& Coleman, 2010) and depression (Dunn et al., 2009). Limited information is available regarding the state of mental health literacy today. This may be due, in part, to internalized stigma about mental illness and the associated lack of awareness.

Poor mental health literacy may be exacerbated by the complexity of the health care and insurance systems in the USA. There are a wide variety of health insurance plans, and all cover different things at different amounts. The health insurance system is complex and is often difficult for consumers to navigate (Consumers Union, 2012). Insurance coverage for behavioral health services plays a role in treatment initiation and completion, given the substantial cost of treatment. Having personal health insurance that provides coverage for behavioral health often leads to higher rates of treatment seeking (Alegría et al. 2012; Lundgren et al., 2005; Pinedo, 2019). Data from the National Survey of Drug Use and Health, an annual, nationally representative, cross-sectional survey, indicates that not only are many individuals un- or under-insured, but many are unaware of, or do not understand, provisions for behavioral health treatment under their plan (Cummings et al., 2014). Given that a part of mental health literacy includes beliefs about the treatability and appropriate course of treatment for mental health concerns, it is likely that there may be some relationship between poor mental health literacy and poor insurance literacy. No study known to the authors has addressed this potential overlap.

Research related to behavioral health help seeking demonstrates that ethnic and racial minority group members are less likely to seek and receive mental health services (Alegria et al., 2012; Drinane et al., 2016; Mennis \& Stahler, 2016). In addition, results of various studies indicate that few African Americans, Latinx Americans, and Asian Americans have sought mental health treatment (Jackson et al., 2007; Le Meyer et al., 2009; Villatoro et al., 2014). What is less well known is how MHL impacts rates of help seeking across group. Few studies have addressed demographic differences in MHL, including those along racial/ ethnic or gender lines. In one study, Vermaas et al. (2017) found no differences in mental 
health literacy rates among clergy of various denominations, including historically Black protestant groups. Some research (Hee Yun Lee et al., 2020; Leighton, 2010) found evidence that there are gender differences in MHL, with women having more social support for and awareness of mental health disorders than do men. This dearth of research related to demographic differences in MHL has led to some researchers (Jorm et al., 2006) to call for additional research on the potential variations in MHL and how those differences might impact behavioral health service utilization.

\section{Mental Health Literacy and Disasters}

Poor mental health literacy, and the potentially confounding challenge of poor health insurance literacy, poses problems for adequate mental health awareness, treatment, and care reimbursement. These difficulties become even more problematic in the context of a mass trauma event, such as the COVID-19 pandemic.

Large-scale disasters often result in mass trauma, triggering depression and anxiety, yet few individuals actively seek help following the event. Trauma exposure, including that related to disasters including pandemics, is associated with anxiety and depression (Breslau \& Anthony, 2007; Roberts et al., 2010; Breslau et al., 2003) and functional impairment, such difficulties at work, home, and school (Berenz et al., 2017, 2018). These mental health sequelae are extensive (Galea et al., 2020; Hawryluck et al., 2004; North, 2016) and can lead to prolonged periods of increased psychiatric symptomology including stress, anxiety, and depression (Bolt et al., 2018; Fussell and Lowe, 2014; Labarda et al., 2020; Seto et al., 2019; Sprague et al., 2014). For example, depression rates among a national probability sample in the USA tripled compared to rates measured pre-pandemic (Ettman et al., 2020). A great deal of research indicates low rates of mental health service utilization relative to need among trauma survivors experiencing mental health symptoms (Sayer et al., 2009; Vogt et al., 2011). Among the concerning barriers to treating the trauma-related mental health sequelae following the COVID-19 pandemic are the factors that drive access to care and service utilization, such as mental health literacy.

\section{Outcomes of Poor Mental Health Literacy}

Lack of mental health literacy is problematic because it serves as a barrier to behavioral health intervention, and perhaps most troubling, it perpetuates stigma surrounding mental illness. Taken together, the barrier to behavioral health intervention coupled with perpetuation of stigma serves to compound barriers to treatment, reducing the likelihood that individuals in need would seek and engage in mental health treatment. In a longitudinal, population study (Bonabi et al., 2016), researchers found that low mental health literacy and negative attitudes toward mental health care were associated with lower levels of behavioral health engagement, even when controlling for sociodemographic characteristics. In their metasynthesis of the extant literature on caregivers' mental health literacy, Hurley and colleagues (Hurley et al., 2019) found that poor mental health literacy was a substantial barrier to behavioral health treatment seeking and was associated with financial and knowledge barriers, fear and mistrust of treatment services, and mental health stigma. Given important group differences in trauma-related mental health experiences that highlight the role of social determinants of health and wellbeing (e.g., gender or social role), it is crucial to have a robust appreciation for the factors contributing to treatment access and utilization during an enduring crisis like COVID-19. 


\section{Summary and Research Questions}

It is clear that mental health literacy influences help seeking behaviors and is connected in meaningful ways to social determinants of health. More information about mental health literacy and its correlates is critical. The following exploratory research questions were answered in the present study:

1. What is the baseline of mental health literacy within the sample?

2. In what ways is mental health literacy distributed across the sample, particularly with regard to race, gender, education, and income?

3. How is mental health literacy associated with depression, anxiety, stress, and mental health stigma more than 6 months into the COVID-19 pandemic?

4. How is mental health literacy associated with health insurance literacy?

\section{Methods}

\section{Centiment Survey Platform Data Collection}

Data were collected using an online survey panel provider who recruits participants widely from online social media platforms including Linked in, Facebook, and other social media websites, as well as through targeted advertisements. The research team specified the criteria for the study's sample a priori-participating individuals had to be over 18, a resident of the USA, and able to read English fluently. Each respondent was then verified using multiple methods, combining IP address, device type, screen size, and cookies to ensure that only unique respondents complete the survey and that all completions are done by human respondents, rather than bots. When potential participants decided to participate, they clicked on the Qualtrics survey link provided in the recruitment document through their social media platform(s), reviewed the consent form and provided consent, completed a captcha attention screener, and then began answering survey items. Participants were compensated for their participation through PayPal transfers from the panel provider to the participant directly.

\section{Measures}

\section{Anxiety}

The Generalized Anxiety Disorder scale (GAD) is a seven-item scale used to assess symptoms of generalized anxiety disorder (Spitzer et al., 2006). Items are rated on a four-point Likert scale $(0=$ not at all, $1=$ several days, $2=$ over half the days, $3=$ nearly every day). Items describe the frequency of features of GAD including feelings of nervousness, irritability, and restlessness. Example items include "Over the last two weeks, how often have you felt nervous, anxious or on edge." Scores on the GAD range from 10 to 28, with higher scores indicating more severe symptoms of anxiety and have demonstrated acceptable internal consistency with $\alpha=0.88$. The alpha for GAD was $\alpha=0.88$ at the start of therapy for the total sample and ranged from 0.83 to $\alpha=0.93$ across units and pre- and post-treatment. Cronbach's alpha was good in the current sample at $\alpha=0.94$. 


\section{Depression}

The Major Depression Inventory (MDI; Olsen et al., 2003) is a 10-item scale used to assess the frequency of depression symptoms. Responses are measured across a sixpoint Likert scale $(0=$ at no time, $1=$ some of the time, $2=$ slightly less than half the time, $3=$ slightly more than half the time, $4=$ most of the time, $5=$ all the time). Higher scores indicate higher levels of depression, and scores ranged from 0 being the lowest possible score to 50 being the highest possible score. Items describe features of major depression including feeling low in spirits and losing interest in daily activities. Example questions include "How often in the last two weeks have you felt low in spirits or sad?" Olsen and colleagues report the measure has strong internal consistency, $\alpha=0.90$; in the current study, $\alpha=0.96$.

\section{Perceived Stress Level}

The Perceived Stress Scale (PSS) is a ten-item assessment of perceived stress level (Cohen 1988). The items focus on feelings of stress in the past month including feelings of control, nervousness, irritability, and task management. Example items include "In the last month, how often have you been upset because of something that happened unexpectedly?" Responses are measured on a five-point Likert scale in which $0=$ never, $1=$ almost never, $2=$ sometimes, $3=$ fairly often, and $4=$ very often. Items $4,5,7$, and 8 require reverse coding. Higher scores indicate higher levels of perceived stress, and possible responses range from 0 to 30. Cohen et al. (1983) report Cronbach's alpha ranging from $\alpha=0.84$ to $\alpha=0.86$ and was adequate in the current sample $\alpha=0.79$.

\section{Caregiver Burden}

The Burden Scale for Family Caregivers (BSFC) short version is a ten-item measure that assesses burden participants experience related to their role as a family caregiver (Pendergrass et al., 2018). Participants respond on a three-point Likert scale $(0=$ strongly disagree, $1=$ disagree, 2 =agree, $3=$ strongly agree . Example items include "My life satisfaction has suffered because of the care I provide." Possible scores range from 0 to 30, and higher scores indicate greater perceived caregiving burden. Cronbach's alpha is reported at 0.92 for the overall score (Pendergrass et al., 2018) and was acceptable in the present sample at $\alpha=0.91$.

\section{Internalized Stigma}

The Internalized Stigma of Mental Illness Inventory (ISMI; Boyd Ritsher et al., 2003) is a 29-item self-report measure developed to assess internalization of stigmatizing attitudes toward mental health disorders. The inventory has five subscales: alienation, stereotype endorsement, perceived discrimination, social withdrawal, and stigma resistance. Responses are collected along a four-point Likert scale in which $1=$ strongly disagree, $2=$ disagree, $3=$ agree , and $4=$ strongly agree. Lower scores indicate higher levels of internalized stigma. The scale has been translated into many languages and demonstrates 
adequate construct, discriminant, convergent, and predictive validity across a number of studies (Boyd et al., 2014). Alpha reliability in the present sample was excellent at $\alpha=0.94$.

\section{Mental Health Literacy}

The Mental Health Literacy Scale (MHLS; O’Connor \& Casey, 2015) was adapted for use in the present study. The MHLS includes a series of items, rated on a Likert-style scale, to assess mental health awareness and attitudes. Awareness questions include identification of symptoms of various psychiatric disorders. Attitudinal questions relate to potentially stigmatizing beliefs, such as "If I had a mental illness I would not tell anyone." Several items were modified to adapt spelling and grammar for American English. Items specifically related to the behavioral health system in Australia were removed.

\section{Insurance Literacy}

To assess the construct of health insurance literacy, or the degree to which participants could accurately define health insurance terms, we asked a series of multiple choice and true or false questions. Answer choices included three plausible answers and an "I'm unsure" option for a four-point response scale. Sample items include "Which of the following options best defines 'health insurance premium'? and True or false: If you are enrolled in a certain type of health insurance plan, you can also set up a tax-free Health Savings Account (HSA) to help save for medical expenses." Scores on health insurance literacy questions suggest that literacy was low $(\mathrm{M}=48.35, \mathrm{SD}=7.31)$, as lower scores on the measure represent higher levels of literacy. Possible scores ranged from 22 to 88 . Cronbach's alpha on this measure was acceptable $(\alpha=0.71)$. Internal consistency is not expected to be high, as the measure is a sum of correct answers, rather than a latent indicator.

\section{Participant Demographic Information}

A total of $N=615$ participants completed a survey about their understanding of mental health terminology. The sample was evenly distributed among male $(n=287,47 \%)$, female $(n=321,52 \%)$, and nonbinary individuals $(n=7,1 \%)$. The mean participant age was 45.39 years old $(\mathrm{SD}=17.2, R=18-91)$. Most participants were identified as White ( $n=494,80 \%$ ), while 55 participants $(9 \%)$ were identified as Black, 36 participants $(6 \%)$ identified as Latinx, and 16 (3\%) identified as mixed race. Twenty-six participants (4\%) were identified as Asian, eight participants (1\%) identified as American Indian or Alaskan native, and two participants $(0.3 \%)$ identified as native Hawaiian. Most participants were identified as married $(n=265,43 \%)$, while $154(25 \%)$ were identified as never married. Some participants reported cohabitating with their partner $(n=56,9 \%)$ or being in a committed relationship $(n=31,5 \%)$, while $61(10 \%)$ were divorced and $15(2 \%)$ were separated. Twenty-nine participants $(5 \%)$ were widowed, and $2(0.3 \%)$ were remarried.

Participants reported varying levels of educational attainment, including those who hold bachelor's degree $(n=151,25 \%)$, attended some college $(n=137,22 \%)$, high school diploma $(n=145,24 \%)$, and those with no formal education $(n=8,1 \%)$. Seventy-two participants $(12 \%)$ held a master's degree, while $23(4 \%)$ held a doctoral or professional degree, and seven (1\%) held a postsecondary non-degree award. Most participants in this sample $(n=263,43 \%)$ held full time employment, while $74(12 \%)$ were employed part time and 127 (21\%) were retired. Though unemployed at the time of this study, some were 
seeking work $(n=64,10 \%)$, while others reported not seeking work $(n=53,9 \%)$. Some participants $(n=33,5 \%)$ were collecting fiscal support at the time of this study. Most participants in the present study were identified as having a relatively low income, with 156 participants $(25 \%)$ earning below \$25,000 annually and $157(26 \%)$ earning between $\$ 25,000$ and $\$ 50,000$ annually. 109 participants (18\%) earned between $\$ 50,000$ and $\$ 75,000$ annually. Few participants $(n=56,9 \%)$ earned more than $\$ 150,000$ per year.

Participants were asked to report whether they were the caregiver of a minor child residing in their home. Less than half of respondents $(n=250,40.7 \%)$ reported that they were a caregiver. Participants were also asked if they had ever been diagnosed with a behavioral health disorder. One-third $(n=190,30.9 \%)$ of respondents reported that they had ever been diagnosed with a mental illness. Only those who were caregivers responded to the BSFC, while only those who had a mental illness diagnosis responded to the ISMI measure (Table 1).

The purpose of this study is to provide information about mental health literacy, mental health, and insurance literacy in a diverse sample of adults in the USA. To analyze research questions, a variety of methods were used so that analysis was consistent with the data type, level of measurement, and analytic approach. First, information about the distribution of data is provided through sharing descriptive statistics related to each measure. Next, information about the association between measures is provided in the reporting of correlations that provide information about the shared variance in each construct. Finally, demographic differences among groups with regard to the measures of mental health literacy, mental health, and health insurance literacy are provided. These tests were conducted using analysis of variance as the goal was to determine whether group differences were present.

\section{Results}

\section{Descriptive Statistics}

Scores on the GAD7, a measure of anxiety, suggested that rates of anxiety were mild $(M=6.16, S D=5.06)$, with scores ranging from 5 to 10 representative of mild anxiety and higher scores indicating more distress. Scores had a strong positive skew, suggesting that the modal score is below the mean. This is an indication that, while the majority of individuals report little anxiety, some are extremely anxious, resulting in a skewed distribution of scores.

Scores on the MDI, a measure of depression, indicated low levels of depression in the sample $(M=17.58, \mathrm{SD}=15.33)$. Scores of less than 20 are generally considered to be below the threshold for clinically significant depression. Scores had a strong positive skew.

The PSS scores, which reflect overall ratings of stress, suggested that the sample were somewhat stressed $(\mathrm{M}=18.12, \mathrm{SD}=6.95)$. Higher scores are representative of more stress. Scores were grouped around the mean.

Scores on a measure of caregiver stress, the BSFC, suggested that caregiver stress levels were moderate $(\mathrm{M}=14.93, \mathrm{SD}=7.40)$ for the 250 individuals who indicated that they were the caregiver for at least one minor child residing in their home. Higher scores are representative of more caregiver burden. Scores had a negative skew, suggesting the mode is higher than the mean.

With regard to mental health literacy, scores suggest that literacy was low $(M=48.35$, $\mathrm{SD}=7.31$ ), as lower scores on the measure represent higher levels of literacy. Possible 
Table 1 Participant demographic information

\begin{tabular}{|c|c|c|}
\hline Characteristic & $n$ & $\%$ \\
\hline \multicolumn{3}{|l|}{ Ethnicity } \\
\hline White & 494 & $80 \%$ \\
\hline Black & 55 & $9 \%$ \\
\hline Latinx & 36 & $6 \%$ \\
\hline Mixed race & 16 & $3 \%$ \\
\hline Asian & 26 & $4 \%$ \\
\hline American Indian & 8 & $1 \%$ \\
\hline Native Hawaiian & 2 & $0.3 \%$ \\
\hline \multicolumn{3}{|l|}{ Gender identity } \\
\hline Female & 321 & $52 \%$ \\
\hline Male & 287 & $47 \%$ \\
\hline Nonbinary & 7 & $1 \%$ \\
\hline \multicolumn{3}{|l|}{ Relationship status } \\
\hline Married & 265 & $43 \%$ \\
\hline Never married & 154 & $25 \%$ \\
\hline Cohabitating & 56 & $9 \%$ \\
\hline Committed relationship & 31 & $5 \%$ \\
\hline Divorced & 61 & $10 \%$ \\
\hline Separated & 15 & $2 \%$ \\
\hline Widowed & 29 & $5 \%$ \\
\hline Remarried & 2 & $0.3 \%$ \\
\hline \multicolumn{3}{|l|}{ Education } \\
\hline Bachelor's degree & 151 & $25 \%$ \\
\hline Some college & 137 & $22 \%$ \\
\hline High school diploma & 145 & $24 \%$ \\
\hline No formal education & 8 & $1 \%$ \\
\hline Master's degree & 72 & $12 \%$ \\
\hline Doctoral or professional degree & 22 & $4 \%$ \\
\hline Postsecondary non-degree award & 7 & $1 \%$ \\
\hline \multicolumn{3}{|l|}{ Income source } \\
\hline Full time employment & 263 & $43 \%$ \\
\hline Part time employment & 74 & $12 \%$ \\
\hline Retired & 127 & $21 \%$ \\
\hline Unemployed, seeking work & 64 & $10 \%$ \\
\hline Unemployed, not seeking work & 53 & $9 \%$ \\
\hline Fiscal assistance/subsidy & 33 & $5 \%$ \\
\hline \multicolumn{3}{|l|}{ Household income } \\
\hline$\$ 25,000$ & 156 & $25 \%$ \\
\hline$\$ 25,000-\$ 50,000$ & 157 & $26 \%$ \\
\hline$\$ 50,000-\$ 75,000$ & 109 & $18 \%$ \\
\hline$\$ 75,000-\$ 100,000$ & 71 & $12 \%$ \\
\hline$\$ 100,000-\$ 125,000$ & 43 & $7 \%$ \\
\hline$\$ 125,000-\$ 150,000$ & 23 & $4 \%$ \\
\hline$\$ 150,000-\$ 175,000$ & 19 & $3 \%$ \\
\hline$\$ 175,000-\$ 200,000$ & 12 & $2 \%$ \\
\hline$\$ 200,000$ or more & 25 & $4 \%$ \\
\hline
\end{tabular}


scores ranged from 22 to 88 . Scores on a measure of internalized stigma, the ISMI, indicated that $(\mathrm{M}=81.44, \mathrm{SD}=16.50)$ individuals had fairly low levels of internalized stigma. On this measure, lower scores are indicative of more internalized stigma, and scores ranged from 44 to 116 .

Health insurance literacy, assessed as the number of correct responses to questions about commonly used health insurance terminology. The average score on this questionnaire was 4.92. Responses to the questionnaire are discussed further in a later section of this report (Table 2).

\section{Correlations}

Correlations among study variables were all in the expected directions. Scores on the measure of mental health literacy were highly negatively correlated with scores on the ISMI $(r=-0.403, p<0.01)$ and HIL $(r=-0.436, p<0.01)$. This indicates that individuals who have poor mental health literacy are also likely to have poor health insurance literacy and more internalized stigma, which all impede access to prompt care for mental health concerns. Scores on the MHL were positively correlated with scores on the GAD $(r=0.212$, $p<0.01)$, MDI $(r=0.237, p<0.01)$, PSS $(r=0.225, p<0.01)$, and BSFC $(r=0.199$, $p<0.05)$. These correlations suggest that those with poorer mental health literacy also experience elevated rates of depression, anxiety, stress, and caregiver burden (Table 3).

Table 2 Means and standard deviations of study variables

\begin{tabular}{lrrl}
\hline & $\mu$ & SD & Range \\
\hline GAD7 & 6.76 & 6.06 & 21 \\
MDI & 17.58 & 15.33 & 60 \\
PSS & 18.12 & 6.95 & 37 \\
MHL & 48.35 & 7.31 & 53 \\
ISMI & 81.44 & 16.50 & 72 \\
HIL & 4.91 & 2.20 & 9 \\
\hline
\end{tabular}

Table 3 Correlations of study variables with MHL scores

\begin{tabular}{|c|c|c|c|c|c|c|c|}
\hline & 1 & 2 & 3 & 4 & 5 & 6 & 7 \\
\hline 1. Anxiety (GAD) & - & & & & & & \\
\hline 2. Depression (MDI) & $0.823 * *$ & - & & & & & \\
\hline 3. Stress (PSS) & $0.707 * *$ & $0.705^{* *}$ & - & & & & \\
\hline 4. Caregiver burden (BSFC) & $0.400 * *$ & $0.494 * *$ & $0.430 * *$ & - & & & \\
\hline 5. Stigma (ISMI) & $-0.299 * *$ & $-481 * *$ & $-0.210 * *$ & $-0.570 * *$ & - & & \\
\hline 6. Health literacy (HIL) & $-0.236 * *$ & $-237 * *$ & $-0.187 * *$ & $-0.138^{*}$ & $0.421 * *$ & - & \\
\hline $\begin{array}{l}\text { 7. Mental health literacy } \\
\text { (MHL) }\end{array}$ & $0.212^{* *}$ & $0.237 * *$ & $0.225 * *$ & $0.199 *$ & $-0.403 * *$ & $-0.436^{* *}$ & - \\
\hline
\end{tabular}

$* *=p<0.01 ; *=p<0.05$ 
Table 4 Frequency of correct response

\begin{tabular}{lll}
\hline & Correct & Incorrect \\
\hline 1. Defining health insurance premium & $387(62.9 \%)$ & $228(37.1 \%)$ \\
2. Defining health insurance deductible & $336(54.6 \%)$ & $279(45.4 \%)$ \\
3. Defining annual out of pocket limit & $399(64.9 \%)$ & $216(35.1 \%)$ \\
4. Defining co-pay & $398(64.7 \%)$ & $217(35.3 \%)$ \\
5. Defining coinsurance & $80(14.6 \%)$ & $525(85.4 \%)$ \\
6. Defining formulary & $157(25.5 \%)$ & $458(74.5 \%)$ \\
7. Defining PCP & $489(79.5 \%)$ & $126(20.5 \%)$ \\
8. Identifying differences in HMO/PPO/POS plans & $255(41.5 \%)$ & $360(58.5 \%)$ \\
9. True/false HSA account purpose & $510(82.9 \%)$ & $105(17.1 \%)$ \\
\hline
\end{tabular}

Table 5 Means and standard deviation of mental health literacy scores by racial group

\begin{tabular}{lll}
\hline & $n$ & $\mu(\mathrm{SD})$ \\
\hline white & 182 & $47.64(7.00)$ \\
Black & 21 & $52.57(9.95)$ \\
Asian & 18 & $52.78(4.54)$ \\
Native American or Pacific & 2 & $42.50(4.95)$ \\
$\quad$ Islander & & \\
"Mixed race" & 5 & $47.80(5.63)$ \\
Latinx & 13 & $46.23(7.06)$ \\
\hline
\end{tabular}

\section{Health Insurance Literacy}

To determine the percentage of respondents who correctly identified commonly used health insurance terminology, we tabulated correct answers on each of the insurance literacy questions. Table 4 presents the results of this tabulation. Of the nine questions, six were answered correctly by more than half of respondents. One question, defining "coinsurance," was answered correctly by less than $15 \%$ of respondents. Gaps in knowledge exist, and among the most concerning, many individuals were unable to correctly identify a plan formulary (25.5\%) or know the difference among common plan types (41.5\%). These rates of response are similar to those found in previous work.

\section{Demographic Differences}

To determine the ways in which mental health literacy was associated with demographic variables, a variety of tests were conducted, based on the type of independent variable. First, significant differences were noted in insurance literacy by race $[\mathrm{F}(6,235)=3.12$, $p<0.01]$. Due to small cell sizes, post hoc testing was not conducted, but means and standard deviations were examined and are presented in Table 5. High scores on the measure are representative of lower mental health literacy, so scores suggest that Black and Asian respondents had the least mental health literacy.

Differences in mental health literacy by education level, income level, and gender were not significant. There were no differences in internalized stigma rates reported by gender, race, or education level. Scores on the internalized stigma measure were, however, different 
across income groups $[\mathrm{F}(8,179)=2.07, p<0.05]$. Post hoc testing using the Scheffee test indicated small difference between the lowest income group (less than $\$ 25,000$ annually) and the highest income groups, though results were not indicative of a direct comparison.

\section{Discussion}

The present study provides information about mental health literacy in a sample of adults in the USA collected during the COVID-19 pandemic. Results suggest that mental health literacy is poor, and individuals are not readily able to identify mental health symptoms and appropriate courses of treatment. Furthermore, mental health literacy is associated with key variables in the expected ways, specifically, that individuals who report poor mental health literacy also report higher rates of depression, anxiety, stress, and internalized stigma. These results indicate troubling low rates of mental health literacy with steep associations noted among internalized stigma and health insurance literacy; however, individual mental health symptoms (e.g., depression) reported by this sample during the COVID-19 pandemic were low compared to other reports from 2020 (Ettman et al. 2020). This discrepancy may be explained by measurement differences, or assessment at different time points. The Ettman et al. (2020) data were collected using the PHQ9 from March to April 2020, compared to the current study, which assessed depression symptoms using the MDI a number of months later, in December 2020. There is evidence that the PHQ9 is an adequate screening instrument but a poor diagnostic tool for measuring symptom severity on its own (Levis et al., 2019; Wittkampf et al., 2009). The MDI, however, is useful both as a reliable measure of depression symptomology but as a screening tool for depression severity (Olsen et al., 2003). Additionally, assessing mental health symptoms in the context of COVID-19 in the first few weeks of the pandemic is considerably different from doing so at the end of the calendar year, by which point the Federal government had articulated the first coordinated response to the virus, including a systematic plan to disseminate vaccines that had not been developed the previous Spring.

The results indicating extremely poor mental health literacy are not surprising as mental health literacy includes ratings of one's ability to recognize the symptoms of mental health difficulties. A sample of responses that includes more variability in mental health literacy, in particular, may evidence different patterns of associations with symptoms reports, as individuals with better mental health literacy may be better able to recognize features of their mental experiences differently. Findings from the present study point to the importance of intervention, especially during disasters when stress is high. Poor mental health literacy is especially problematic when large swathes of the population are struggling with mental health concerns, including depression and anxiety (BLINDED). The public faces the daunting challenge of making sense of the tremendous volume of COVID-19 information available across a wide range of information platforms as they learn about the virus while facing the reality of unprecedented uncertainty (Abel \& McQueen, 2020). This is undoubtedly challenging, and mental health literacy may impact the facility with which individuals can take in critical health information.

Impacts of poor mental health literacy are felt acutely for caregivers (Hurley et al., 2019). Parents not only report difficulty identifying when to intervene with children who are struggling (Boulter \& Rickwood, 2013), but also report high levels of burden 
associated with the caregiving role during periods of heightened stress like the COVID19 pandemic (BLINDED). Results from the present study certainly support associations between poor mental health literacy and caregiver burden. These associations underscore the need for interventions that target parents of minors to specifically bolster caregivers' abilities to recognize psychological distress in those they care for with the aim of improving treatment engagement for children who rely on caregivers to activate care on their behalf.

Finally, results point to preliminary evidence that suggests that mental health literacy is differentially distributed across racial groups. This provides some evidence to suggest that mental health literacy may be impacted by social determinants of health and, may be a powerful barrier to service utilization for mental health concerns.

\section{Limitations}

Despite the utility of the findings, this study is not without its limitations. First, the use of a paneling company poses a challenge to external validity. While the sample is a national sample, it is not a probability sample and therefore generalizability is limited. Furthermore, analyses of differences in mental health literacy by age, income, and education were not significant. This is perhaps due to those analyses being under-powered. Larger samples with more diversity in response are required to ensure adequate power for multiple group testing. Furthermore, additional inquiries into the link between social determinants of health and mental health literacy are necessary. More samples are needed, perhaps using GIS indicators of location to better align features of disparity by place rather than solely relying on individual characteristics reported by the respondents. Finally, the present study was cross-sectional in nature, and there are several limitations of the methodology used. Cross-sectional designs, while useful for exploratory research such as this, are limited in their ability to determine direction of association, or causal elements. More direct assessment of association is warranted, and future researchers may wish to consider utilizing a MHL intervention to begin the work of remedying poor MHL and assessing the outcomes of that approach.

\section{Conclusion}

The present study provides powerful evidence that mental health literacy is poor and is associated with symptoms of depression and anxiety, as well as higher levels of stress, more internalized stigma about mental illness, and higher ratings of caregiver burden for those caring for children during the COVID-19 pandemic. Mental health illiteracy is a barrier to behavioral health treatment seeking, both for individuals themselves and for those for whom they provide care. The deleterious outcomes of poor mental health literacy, and poor mental health in general, may be exacerbated during times of stress, such as a global pandemic. Intervention is critical, and mental health literacy intervention may be one key way to improve behavioral health outcomes for individuals and families. 


\section{Declarations}

The study and all protocol were reviewed and approved by the University of Connecticut Institutional Review Board, protocol X20-0211.

Conflict of Interest The authors have no potential conflicts of interest to report.

Informed Consent Informed consent was obtained from all participants.

\section{References}

Abel, T., \& McQueen, D. (2020). Critical health literacy and the COVID-19 crisis. Health Promotion International, 35.https://doi.org/10.1093/heapro/daaa040.

Alegria, M., Lin, J., Chen, C.-N., Duan, N., Cook, B., \& Meng, X.-L. (2012). The impact of insurance coverage in diminishing racial and ethnic disparities in behavioral health services. Health Services Research, 47(3), 1322-1344. https://doi.org/10.1111/j.1475-6773.2012.01403.x

Berenz, E. C., Roberson-Nay, R., Latendresse, S. J., Mezuk, B., Gardner, C. O., Amstadter, A. B., \& York, T. P. (2017). Posttraumatic stress disorder and alcohol dependence: Epidemiology and order of onset. Psychological Trauma: Theory, Research, Practice, and Policy, 9(4), 485-492. https://doi.org/10. 1037/tra0000185

Berenz, E. C., York, T. P., Bing-Canar, H., Amstadter, A. B., Mezuk, B., Gardner, C. O., \& Roberson-Nay, R. (2018). Time course of panic disorder and posttraumatic stress disorder onsets. Social Psychiatry and Psychiatric Epidemiology, 54(5), 639-647. https://doi.org/10.1007/s00127-018-1559-1

Bolt, M. A., Helming, L. M., \& Tintle, N. L. (2018). The associations between self-reported exposure to the Chernobyl nuclear disaster zone and mental health disorders in Ukraine. Frontiers in Psychiatry, 9.https://doi.org/10.3389/fpsyt.2018.00032.

Bonabi, H., Müller, M., Ajdacic-Gross, V., Eisele, J., Rodgers, S., Seifritz, E., Rössler, W., \& Rüsch, N. (2016). Mental health literacy, attitudes to help seeking, and perceived need as predictors of mental health service use. The Journal of Nervous and Mental Disease, 204(4), 321-324. https://doi.org/10. 1097/nmd.0000000000000488

Boulter, E., \& Rickwood, D. (2013). Parents' experience of seeking help for children with mental health problems. Advances in Mental Health, 11(2), 131-142. https://doi.org/10.5172/jamh.2013.11.2.131

Boyd, J. E., Adler, E. P., Otilingam, P. G., \& Peters, T. (2014). Internalized stigma of mental illness (ISMI) scale: A multinational review. Comprehensive Psychiatry, 55(1), 221-231. https://doi.org/10.1016/j. comppsych.2013.06.005

Boyd Ritsher, J., Otilingam, P. G., \& Grajales, M. (2003). Internalized stigma of mental illness: Psychometric properties of a new measure. Psychiatry Research, 121(1), 31-49. https://doi.org/10.1016/j.psych res.2003.08.008

Breslau, N., \& Anthony, J. C. (2007). Gender differences in the sensitivity to posttraumatic stress disorder: An epidemiological study of urban young adults. Journal of Abnormal Psychology, 116(3), 607-611. https://doi.org/10.1037/0021-843x.116.3.607

Breslau, N., Davis, G. C., \& Schultz, L. R. (2003). Posttraumatic stress disorder and the incidence of nicotine, alcohol, and other drug disorders in persons who have experienced trauma. Archives of General Psychiatry, 60(3), 289. https://doi.org/10.1001/archpsyc.60.3.289

Coles, M. E., \& Coleman, S. L. (2010). Barriers to treatment seeking for anxiety disorders: Initial data on the role of mental health literacy. Depression and Anxiety, 27(1), 63-71. https://doi.org/10.1002/da.20620

Consumers Union. (2012). Measuring health insurance literacy: A call to action. http://consumersunion.org/ pub/Health_Insurance_Literacy_Roundtablerpt.pdf

Cohen, S. (1988). Perceived stress in a probability sample of the United States. In S. Spacapan \& S. Oskamp (Eds.), The social psychology of health. (1988-98838-002; pp. 31-67). Sage Publications, Inc; APA PsycInfo. https://ezproxy.lib.uconn.edu/login?url=https://search.ebscohost.com/login.aspx?direct=true $\& \mathrm{db}=$ psyh\&AN=1988-98838-002\&site=ehost-live

Cohen, S., Kamarck, T., \& Mermelstein, R. (1983). A global measure of perceived stress. Journal of Health and Social Behavior, 24(4), 385-396. APA PsycInfo. https://doi.org/10.2307/2136404

Cummings, J. R., Wen, H., Ritvo, A., \& Druss, B. G. (2014). Health insurance coverage and the receipt of specialty treatment for substance use disorders among US adults. Psychiatric Services, 65(8), 10701073. https://doi.org/10.1176/appi.ps.201300443 
Drinane, J. M., Owen, J., \& Kopta, S. M. (2016). Racial/ethnic disparities in psychotherapy: Does the outcome matter? In TPM-Testing, Psychometrics, Methodology in Applied Psychology (Vol. 23). https:// ezproxy.lib.uconn.edu/login?url=https://search.ebscohost.com/login.aspx?direct=true \&db=psyh\& AN=2017-11753-008\&site=ehost-live

Dunn, K. I., Goldney, R. D., Grande, E. D., \& Taylor, A. (2009). Quantification and examination of depression-related mental health literacy. Journal of Evaluation in Clinical Practice, 15(4), 650-653. https:// doi.org/10.1111/j.1365-2753.2008.01067.x

Ettman, C. K., Abdalla, S. M., Cohen, G. H., Sampson, L., Vivier, P. M., \& Galea, S. (2020). Prevalence of depression symptoms in US adults before and during the COVID-19 pandemic. JAMA Network Open, 3, e2019686. https://doi.org/10.1001/jamanetworkopen.2020.19686

Fussell, E., \& Lowe, S. R. (2014). The impact of housing displacement on the mental health of low-income parents after Hurricane Katrina. Social Science \& Medicine, 113, 137-144. https://doi.org/10.1016/j. socscimed.2014.05.025

Galea, S., Merchant, R. M., \& Lurie, N. (2020). The mental health consequences of COVID-19 and physical distancing. JAMA Internal Medicine, 180(6), 817. https://doi.org/10.1001/jamainternmed.2020.1562

Hawryluck, L., Gold, W. L., Robinson, S., Pogorski, S., Galea, S., \& Styra, R. (2004). undefined. Emerging Infectious Diseases, 10(7), 1206-1212. https://doi.org/10.3201/eid1007.030703

Hee Yun Lee, Junseon Hwang, Ball, J. G., Jongwook Lee, Youngmi Yu, \& Albright, D. L. (2020). Mental Health Literacy Affects Mental Health Attitude: Is There a Gender Difference? American Journal of Health Behavior, 44(3), 282-291. Academic Search Premier.

Hurley, D., Swann, C., Allen, M. S., Ferguson, H. L., \& Vella, S. A. (2019). A systematic review of parent and caregiver mental health literacy. Community Mental Health Journal, 56(1), 2-21. https://doi.org/ 10.1007/s10597-019-00454-0

Jackson, J. S., Neighbors, H. W., Torres, M., Martin, L. A., Williams, D. R., Baser, R., \& Lowe, J. (2007). Use of mental health services and subjective satisfaction with treatment among Black Caribbean immigrants: Results from the National Survey of American Life. American Journal of Public Health, 97(1), 60-67. APA PsycInfo. https://doi.org/10.2105/AJPH.2006.088500

Jorm, A. F. (2015). Why we need the concept of "mental health literacy." Health Communication, 30(12), 1166-1168. https://doi.org/10.1080/10410236.2015.1037423

Jorm, A. F., Barney, L. J., Christensen, H., Highet, N. J., Kelly, C. M., \& Kitchener, B. A. (2006). Research on mental health literacy: What we know and what we still need to know. Australian \& New Zealand Journal of Psychiatry, 40(1), 3-5. https://doi.org/10.1080/j.1440-1614.2006.01734.x

Kessler, R. C., Berglund, P. A., Dewit, D. J., Bedirhan Üstün, T., Wang, P. S., \& Wittchen, H. (2002). Distinguishing generalized anxiety disorder from major depression: Prevalence and impairment from current pure and comorbid disorders in the US and Ontario. International Journal of Methods in Psychiatric Research, 11(3), 99-111. https://doi.org/10.1002/mpr.128

Kessler, R. C., Petukhova, M., Sampson, N. A., Zaslavsky, A. M., \& Wittchen, H. (2012). Twelve-month and lifetime prevalence and lifetime morbid risk of anxiety and mood disorders in the United States. International Journal of Methods in Psychiatric Research, 21(3), 169-184. https://doi.org/10.1002/ mpr.1359

Labarda, C. E., Jopson, Q. D., Hui, V. K., \& Chan, C. S. (2020). Long-term displacement associated with health and stress among survivors of typhoon Haiyan. Psychological Trauma: Theory, Research, Practice, and Policy, 12(7), 765-773. https://doi.org/10.1037/tra0000573

Levis, B., Benedetti, A., Thombs, B. D., \& DEPRESsion Screening Data (DEPRESSD) Collaboration,. (2019). Accuracy of patient health questionnaire-9 (PHQ-9) for screening to detect major depression: Individual participant data meta-analysis. BMJ (Clinical Research Ed.), 365, 11476. https://doi.org/10. 1136/bmj.11476

Le Meyer, O., Zane, N., Cho, Y. I., \& Takeuchi, D. T. (2009). Use of specialty mental health services by Asian Americans with psychiatric disorders. Journal of Consulting and Clinical Psychology, 77(5), 1000-1005. APA PsycInfo. https://doi.org/10.1037/a0017065

Leighton, S. (2010). Using a vignette-based questionnaire to explore adolescents' understanding of mental health issues. Clinical Child Psychology and Psychiatry, 15(2), 231-250. https://doi.org/10.1177/ 1359104509340234

Lundgren, L. M., Amaro, H., \& Ben-Ami, L. (2005). Factors Associated with Drug Treatment Entry Patterns Among Hispanic Women Injection Drug Users Seeking Treatment. Journal of Social Work Practice in the Addictions, 5(1-2), 157-174. https://doi.org/10.1300/J160v5n01_08

Mendenhall, A. N., \& Frauenholtz, S. (2013). Mental health literacy: Social work's role in improving public mental health. Social Work, 58(4), 365-368. https://doi.org/10.1093/sw/swt038 
Mennis, J., \& Stahler, G. J. (2016). Racial and ethnic disparities in outpatient substance use disorder treatment episode completion for different substances. Journal of Substance Abuse Treatment, 63, 25-33. https://doi.org/10.1016/j.jsat.2015.12.007

North, C. S. (2016). Disaster mental health epidemiology: Methodological review and interpretation of research findings. Psychiatry, 79(2), 130-146. https://doi.org/10.1080/00332747.2016.1155926

Olsen, L. R., Jensen, D. V., Noerholm, V., Martiny, K., \& Bech, P. (2003). The internal and external validity of the major depression inventory in measuring severity of depressive states. Psychological Medicine, $33,351-356$.

O'Connor, M., \& Casey, L. (2015). The Mental Health Literacy Scale (MHLS): A new scale-based measure of mental health literacy. Psychiatry Research, 229(1-2), 511-516. https://doi.org/10.1016/j.psychres. 2015.05.064

Pendergrass, A., Malnis, C., Graf, U., Engel, S., \& Graessel, E. (2018). Screening for caregivers at risk: Extended validation of the short version of the Burden Scale for Family Caregivers (BSFC-s) with a valid classification system for caregivers caring for an older person at home. BMC Health Services Research, 18(1), 229. https://doi.org/10.1186/s12913-018-3047-4

Pinedo, M. (2019). A current re-examination of racial/ethnic disparities in the use of substance abuse treatment: Do disparities persist? Drug and Alcohol Dependence, 202, 162-167. https://doi.org/10.1016/j. drugalcdep.2019.05.017

Roberts, A. L., Gilman, S. E., Breslau, J., Breslau, N., \& Koenen, K. C. (2010). Race/ethnic differences in exposure to traumatic events, development of post-traumatic stress disorder, and treatment-seeking for post-traumatic stress disorder in the United States. Psychological Medicine, 41(1), 71-83. https://doi. org/10.1017/s0033291710000401

Roll, J. M., Kennedy, J., Tran, M., \& Howell, D. (2013). Disparities in unmet need for mental health services in the United States, 1997-2010. Psychiatric Services, 64(1), 80-82. https://doi.org/10.1176/ appi.ps.201200071

Sayer, N. A., Friedemann-Sanchez, G., Spoont, M., Murdoch, M., Parker, L. E., Chiros, C., \& Rosenheck, R. (2009). A qualitative study of determinants of PTSD treatment initiation in veterans. Psychiatry: Interpersonal and Biological Processes, 72(3), 238-255. https://doi.org/10.1521/psyc.2009.72.3.238

Seto, M., Nemoto, H., Kobayashi, N., Kikuchi, S., Honda, N., Kim, Y., Kelman, I., \& Tomita, H. (2019). Post-disaster mental health and psychosocial support in the areas affected by the great east Japan earthquake: A qualitative study. BMC Psychiatry, 19(1). https://doi.org/10.1186/s12888-019-2243-z.

Smith, C. L., \& Shochet, I. M. (2011). The impact of mental health literacy on help-seeking intentions: Results of a pilot study with first year psychology students. International Journal of Mental Health Promotion, 13(2), 14-20. https://doi.org/10.1080/14623730.2011.9715652

Sprague, C. M., Kia-Keating, M., Felix, E., Afifi, T., Reyes, G., \& Afifi, W. (2014). Youth psychosocial adjustment following wildfire: The role of family resilience, emotional support, and concrete support. Child \& Youth Care Forum, 44(3), 433-450. https://doi.org/10.1007/s10566-014-9285-7

Spitzer, R. L., Kroenke, K., Williams, J. B. W., \& Löwe, B. (2006). A brief measure for assessing generalized anxiety disorder: The GAD-7. Archives of Internal Medicine, 166(10), 1092-1097. https://doi.org/ 10.1001/archinte.166.10.1092

Thornicroft, G. (2007). Most people with mental illness are not treated. The Lancet, 370(9590), 807-808. https://doi.org/10.1016/s0140-6736(07)61392-0

Vermaas, J. D., Green, J., Haley, M., \& Haddock, L. (2017). Predicting the Mental Health Literacy of Clergy: An Informational Resource for Counselors. Journal of Mental Health Counseling, 39(3), 225241. Academic Search Premier

Villatoro, A. P., Morales, E. S., \& Mays, V. M. (2014). Family culture in mental health help-seeking and utilization in a nationally representative sample of Latinos in the United States: The NLAAS. American Journal of Orthopsychiatry, 84(4), 353-363. APA PsycInfo. https://doi.org/10.1037/h0099844

Vogt, D., Vaughn, R., Glickman, M. E., Schultz, M., Drainoni, M., Elwy, R., \& Eisen, S. (2011). Gender differences in combat-related stressors and their association with postdeployment mental health in a nationally representative sample of U.S. oef/OIF veterans. Journal of Abnormal Psychology, 120(4), 797-806. https://doi.org/10.1037/a0023452

Wittkampf, K., van Ravesteijn, H., Baas, K., van de Hoogen, H., Schene, A., Bindels, P., Lucassen, P., van de Lisdonk, E., \& van Weert, H. (2009). The accuracy of Patient Health Questionnaire-9 in detecting depression and measuring depression severity in high-risk groups in primary care. General Hospital Psychiatry, 31, 451-459. https://doi.org/10.1016/j.genhosppsych.2009.06.001

Publisher's Note Springer Nature remains neutral with regard to jurisdictional claims in published maps and institutional affiliations. 